\title{
A Comparison of 4D DSA with 2D and 3D DSA in the Analysis of Normal Vascular Structures in a Canine Model
}

\author{
C. Sandoval-Garcia, K. Royalty, B. Aagaard-Kienitz, S. Schafer, (DP. Yang, and C. Strother
}

\begin{abstract}
BACKGROUND AND PURPOSE: 4D DSA allows viewing of 3D DSA as a series of time-resolved volumes of a contrast bolus. There is no comparison of the accuracy of the anatomic information provided by 4D DSA with that available from conventional 2D and 3D DSA. Our purpose was to make this comparison by using a canine model.
\end{abstract}

MATERIALS AND METHODS: 2D, 3D, and 4D DSA acquisitions were performed in 5 canines from 3 catheter positions in the common carotid artery yielding 15 2D, 15 3D, and 15 4D datasets. For each territory, 3 vascular segments were chosen for comparison. Images were reviewed by 2 experienced neuroradiologists and were graded by the ability to visualize a segment, its filling direction, and preferred technique. Two visualization modes for 4D DSA were compared (volume-rendering technique and MIP).

RESULTS: 4D DSA was preferred in $73.9 \%$ of the image sets; $2 \mathrm{D}$, in $22.7 \%$; and 3D, in $3.4 \%$. 4D DSA MIP rendering yielded superior visualization of very small vessel details; the 4D DSA volume-rendering technique offered superior depth and overlap information and better visualization of the surface details of the vasculature.

CONCLUSIONS: In this study, 4D DSA was preferred over 2D and 3D DSA for analysis of normal vasculature. The ability to provide any view of a vascular territory at any time during passage of a contrast bolus seems likely to reduce the need for many 2D acquisitions during diagnostic and therapeutic procedures. This then potentially translates into a reduction in radiation and contrast dose.

ABBREVIATION: VRT = volume-rendering technique

4 D DSA provides the ability to create a series of time-resolved volumes of vasculature so that the passage of a contrast bolus may be viewed in 3D at any time and from any angle. Traditional 3D DSA acquisitions provide volumetric anatomic information but are not time-resolved. The overlap of vasculature in these 3D images often makes it difficult to analyze details (eg, angioarchitecture of an AVM nidus). To overcome this problem, multiple 2D DSA acquisitions at different angles are often necessary. The $4 \mathrm{D}$ algorithm applied to data from a rotational acquisition, obtained by using an injection protocol that starts the injection shortly after rotation of the C-arm rather than

Received November 11, 2014; accepted after revision February 16, 2015

From the Departments of Neurological Surgery (C.S.-G., B.A.-K.), Biomedical Engineering (K.R), and Radiology (P.Y., C.S.), University of Wisconsin School of Medicine and Public Health, Madison, Wisconsin; Siemens (K.R., S.S.), Hoffman Estates, Illinois; and Department of Neurosurgery (P.Y.), Changhai Hospital, Second Military Medical University, Shanghai, China.

This work was supported by National Institutes of Health grant R01 144-PRJ68DN.

Please address correspondence to Charles Strother, MD, Department of Radiology, University of Wisconsin School of Medicine and Public Health, E3/366 Clinical Sciences Center, 600 N Highland Ave, Madison, WI 53792; e-mail: strother@wisc.edu

- Indicates open access to non-subscribers at www.ajnr.org

http://dx.doi.org/10.3174/ajnr.A4359 simultaneous with its rotation, results in a series of 4D DSA volumes that provides a user with the ability to view both anatomic information and contrast dynamics. ${ }^{1}$ The anatomic information provided by this technique has not, to our knowledge, been compared with that provided by conventional 2D and 3D DSA images. In this study, we aimed to assess the ability of 4D DSA to depict vascular anatomy. Our methods also aimed to acquire data that would allow us to make judgments as to whether the content was superior to and/or complementary to that of conventional DSA studies. We believed that this comparison was important because the ability to view relevant vasculature at any time and at any angle with a $4 \mathrm{D}$ reconstruction should result in less need to acquire multiple 2D series in both diagnostic and interventional procedures, thereby leading to reductions in both $\mathrm{x}$ ray and contrast medium doses.

\section{MATERIALS AND METHODS}

2D, 3D, and 4D DSA images of 3 matching vascular territories from 5 canines were obtained by using a commercially available biplane angiography system (Artis zee; Siemens, Erlangen, Germany). All studies were performed under a protocol approved by the University of Wisconsin Animal Care Committee. 


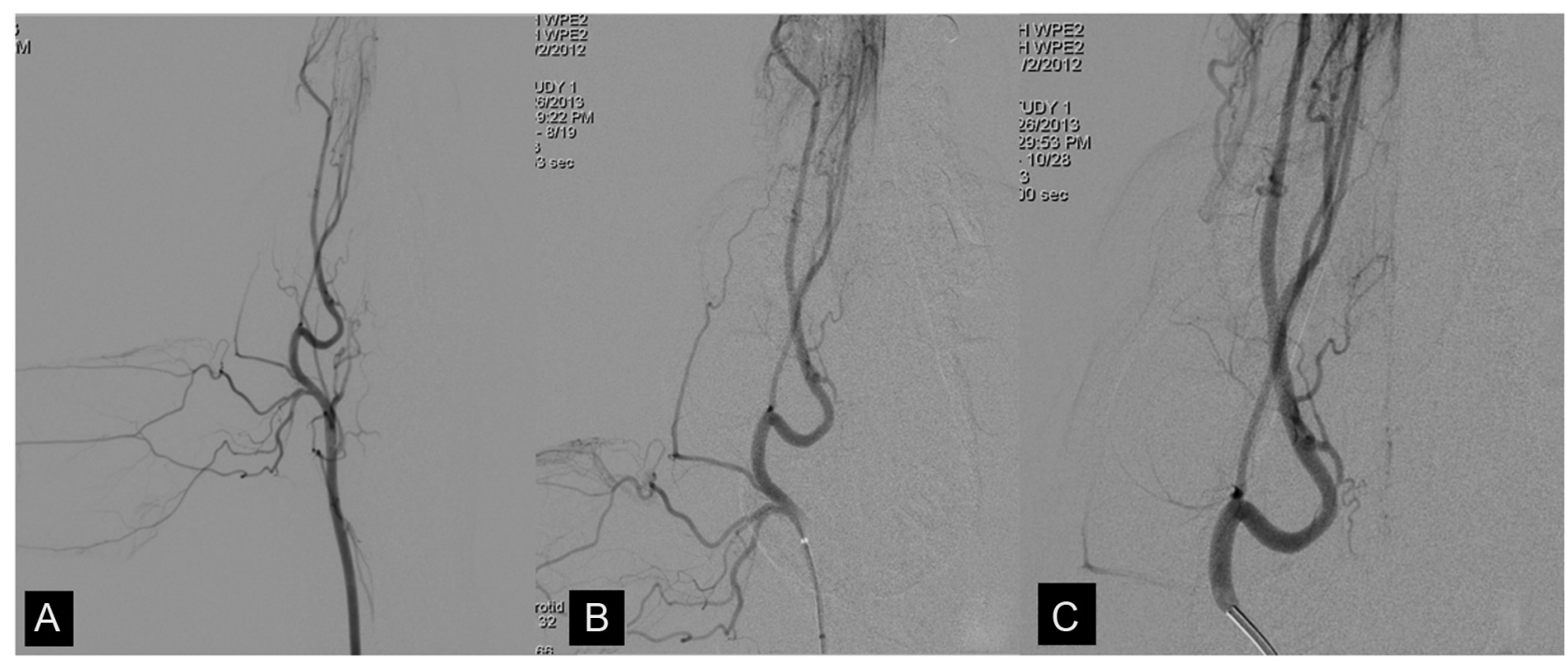

FIG 1. Example of the vasculature on anteroposterior 2D DSA projections for each catheter position: proximal $(A)$, middle ( $B)$, and distal $(C)$. These show the decrease in the vascular complexity as the injection site is moved from proximal to most distal. The magnification factor for the images increases, moving from proximal to most distal.

Table 1: Injection parameters used for the various acquisitions

\begin{tabular}{|c|c|c|c|c|c|c|c|c|c|}
\hline & \multicolumn{3}{|c|}{ Proximal } & \multicolumn{3}{|c|}{ Middle } & \multicolumn{3}{|c|}{ Distal } \\
\hline & 2D DSA & 3D DSA & 4D DSA & 2D DSA & 3D DSA & 4D DSA & 2D DSA & 3D DSA & 4D DSA \\
\hline Contrast volume (mL) & $8-9$ & 20 & 20 & $8-9$ & 11 & 11 & $8-9$ & 8 & 9 \\
\hline Concentration (\%) & 50 & 50 & 50 & 50 & 50 & 50 & 50 & 50 & 50 \\
\hline Flow rate $(\mathrm{mL} / \mathrm{s})$ & $\mathrm{HI}$ & 3 & 3 & $\mathrm{HI}$ & $1.5-2.5$ & $1.5-2.5$ & $\mathrm{HI}$ & $1-1.5$ & $1-1.5$ \\
\hline Injection duration (s) & $\mathrm{HI}$ & 6.6 & 6.6 & $\mathrm{HI}$ & 6 & 6 & $\mathrm{HI}$ & 7.3 & 7.3 \\
\hline Injection delay (s) & 1 & 0 & 0 & 1 & 0 & 0 & 1 & 0 & 0 \\
\hline X-ray delay (s) & 0 & 1 & 0 & 0 & 1 & 0 & 0 & 1 & 0 \\
\hline
\end{tabular}

Note:- HI indicates hand injection.

\section{Imaging Acquisition}

After induction of endotracheal anesthesia, a $4 \mathrm{~F}$ catheter was introduced into 1 common femoral artery. Under fluoroscopic guidance, the catheter was positioned first in one of the common carotid arteries, then more distally at a location just past the origin of the ICA, and finally still more distally at a location just beyond the superficial temporal artery. This sequence of locations for injections was chosen so that there would be a decrease in the complexity of the vasculature as the injection location was moved more distally. Figure 1 shows an example of these vascular territories on 2D DSA images.

The injection protocol rate, volume, contrast concentration, and timing were aimed at minimizing the reflux of contrast and optimizing enhancement of the vessels for a given acquisition. A dual-syringe power injector (Accutron; Medtron, Saarbrücken, Germany) was used for all power injections. To mimic the bolus characteristics of a clinical study, we performed the 2D DSAs by using a hand injection of the contrast medium. Table 1 summarizes the injection protocols.

The 2D DSA images were acquired in standard anteroposterior and lateral projections. A conventional 3D DSA acquisition protocol was used to acquire a subtracted $3 \mathrm{D}$ dataset $(5$ seconds, $200^{\circ}$ ). For a typical 3D DSA clinical acquisition protocol, rotation of the C-arm is started 1-2 seconds after injection of the contrast to allow the contrast to reach a more steady-state of vascular opacification before the start of data acquisition. Because the aim of the $4 \mathrm{D}$ technique is to capture both anatomic and temporal information of the contrast dynamics to capture the inflow of contrast, the $\mathrm{C}$-arm rotation is started shortly $(0.5-1$ second) before injection of the contrast. To capture the steadystate and because of the rapid circulation time in the canine, we acquired the $4 \mathrm{D}$ datasets by using a 6 -second $260^{\circ}$ acquisition program.

\section{Image Reconstruction}

All 2D, 3D, and 4D DSA projection data were transferred to a research workstation running both commercial software (syngo X-Workplace VB21; Siemens) and 4D DSA prototype software. The 3D DSA reconstructions were performed by using the product software and standard reconstruction kernels (HU auto) with a projection-based motion-correction algorithm applied to minimize artifacts from any subject motion during the acquisition.

Once the 3D DSA reconstruction was complete, the 4D DSA reconstruction was performed. The $4 \mathrm{D}$ DSA reconstruction is a 2-step process; first, a conventional 3D DSA constraint volume is reconstructed by using the product software and the same reconstruction parameters used for the $3 \mathrm{D}$ reconstruction described above. Following this step, the temporal information contained in the rotational projection sequence is encoded into the constraint volume for every projection image. This process effectively creates a temporal volumetric representation of the contrast flow 


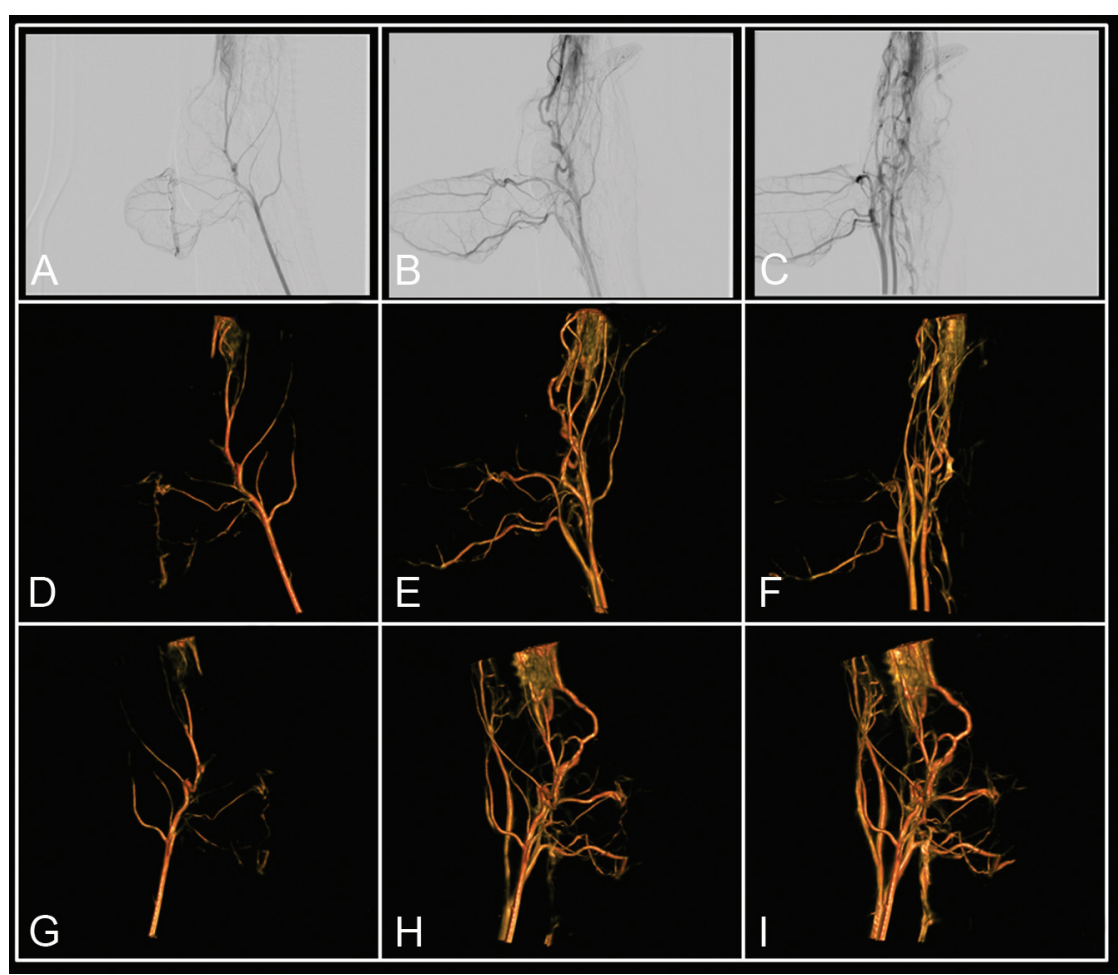

FIG 2. Illustration of $4 D$ DSA reconstruction. No x-ray delay between contrast injection and rotational image acquisition results in contrast flow or inflow being visible in the rotational projections $(A-C)$. Through a 2-step reconstruction process, this flow information is encoded into the 3D DSA for every projection, effectively creating a 4D DSA. This allows viewing of the contrast bolus passage at any desired angle at any time during the bolus passage. $D-F$, View of the bolus passage at 3 projection angles at 3 different time points. $G-I$, View of the bolus passage at 3 angles not present in the projections, again at 3 different time points. The figures correspond to sample angle projections selected to match the 2D acquisitions, even though once 4D images are reconstructed, views are available from any angle.

through the subject's vasculature at the frame rate of the rotational projection image acquisition (typically 30 frames/s). ${ }^{1}$ Figure 2 shows an example of the contrast dynamics in the rotational projections along with a display of these dynamics as seen in the $4 \mathrm{D}$ reconstructions.

\section{Image Evaluation}

Three vascular segments were selected on each of the 2D image datasets. The same segments were used on the $2 \mathrm{D}$ studies from each of the 5 canines. Annotations were placed indicating the location of each segment. Scoring forms and labeled images were provided to the reviewers with a pictorial reference to clearly identify which vascular segments were to be evaluated. Figure $3 A,-B$ shows an example of the material that was provided to the reviewers. The vessel characteristics that were evaluated included clarity of segment extent and margins, evidence of superimposed or adjacent vessels obscuring visualization, direction of filling, and individual rating of each of the available display modalities for 4D DSA and the choice of the overall preferred image. The Hinkmann rating scale (1-5) was used because it has been used for similar image evaluation tasks in previously published literature. ${ }^{2}$ The reviewer was also asked to score the image quality of the 4D DSA (with respect to a given vascular segment) for 2 different rendering schemes (volume-rendering technique [VRT] and MIP), and a separate scale from 0 to 4 was used for this portion of the review. Both scales are shown in Fig $3 B$.
Each observer reviewed the image sets separately. All images were viewed on a workstation, and observers were allowed to manipulate all images (review, window, stop, magnify, and so forth) as they would do in clinical practice.

The reviewer was first presented with the 2D DSA image sequences and asked to score the 3 vascular segments from each territory ( 9 segments for each of the 5 canines). Next, the observer was shown the 3D DSA reconstructions of the corresponding territories and was asked to score the same 9 segments. Finally, the 4D DSA reconstruction was presented, and the same evaluation procedure was repeated. The reviewer was also asked to evaluate each of the 4D DSA studies when displayed either as a surface-rendered (VRT) volume or as an MIP and was allowed to make comments about any of the image datasets. The scoring schemes used for each of the evaluations are shown in Fig 3.

The 2 observers independently evaluated 44 vessel segments from the $5 \mathrm{ca}$ nines ( 1 canine only had 8 vessel segments included for evaluation because 1 segment was not adequately visualized in any technique).

\section{RESULTS}

Overall, the 2 observers preferred the 4D DSA in $73.9 \%$ of the 88 image sets. The 2D DSA images were preferred in $22.7 \%$ of the image sets; the 3D DSA images were preferred in only $3.4 \%$. Table 2 shows the raw data scores of each reviewer.

As can be seen from the raw data scores, one of the observers preferred the $4 \mathrm{D}$ images much more often than did the other $4 \mathrm{D}$ preferred in 40 of the 44 [91\%] datasets by observer 2 compared with 25 of 44 [57\%] by observer 1). These data also show that in 12 of the $28(43 \%)$ image sets in which observer 1 preferred the $2 \mathrm{D}$ images, both the $2 \mathrm{D}$ and $4 \mathrm{D}$ images were, nonetheless, scored equally (ie, 4, excellent image quality).

Both 4D VRT and 4D MIP rendering yielded the same average rating of 1.9 , in which 1 represented excellent image quality. In general, observers thought that the MIP rendering yielded superior visualization of very small vessel details, and the VRT offered superior depth and overlap information and better visualization of the surface details of the vasculature.

\section{DISCUSSION}

4D DSA provides a means to acquire a fully time-resolved 3D reconstruction of a vascular territory having superior spatial and temporal resolution to any other commercially available $4 \mathrm{D}$ imaging technique. ${ }^{1}$ In this small study, we have shown that for the evaluation of the extracranial canine vasculature, $4 \mathrm{D}$ 

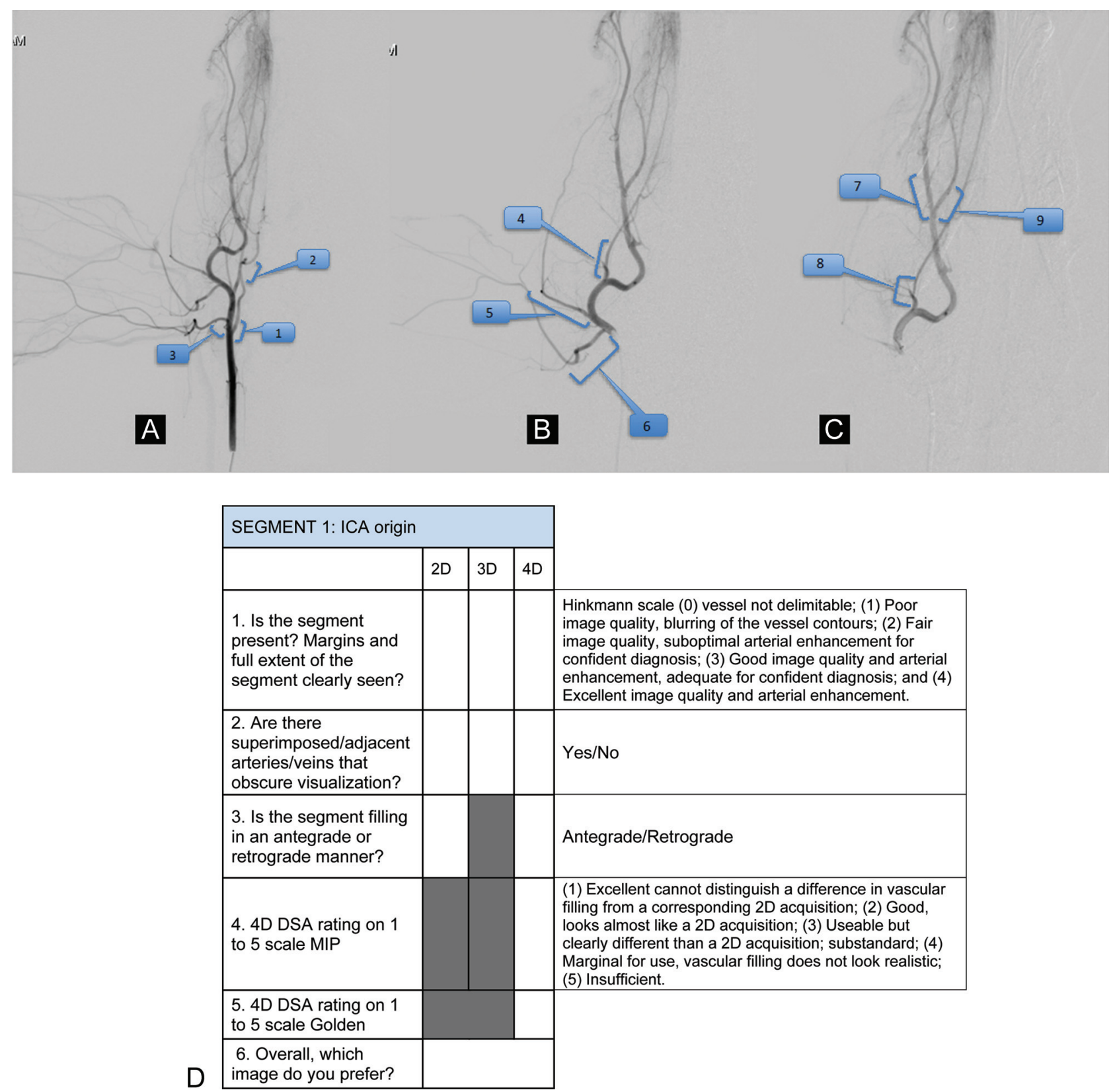

FIG 3. $A-C$, Example of the pictorial reference form provided to the image reviewers identifying the vascular segments to be evaluated: proximal $(A)$, middle $(B)$, and distal $(C)$ catheter positions on anteroposterior projections. $D$, Evaluation form and scales used for scoring of the images.

DSA frequently provided information that 2 experienced observers preferred over that provided by $2 \mathrm{D}$ or $3 \mathrm{D}$ studies. Although we did not explicitly ask whether the 4D dataset contained information equivalent to that in the combination of $2 \mathrm{D}$ and $3 \mathrm{D}$ series, we believe that this may be the case. All experience to date indicates that the $4 \mathrm{D}$ reconstruction contains the same information as a conventional $3 \mathrm{D}$ reconstruction and the time-resolved information contained in a full "library" of unlimited 2D acquisitions. The ability to view the vasculature at any desired angle and at any time during passage of a contrast bolus should allow elimination of most of the $2 \mathrm{D}$ acquisitions that now are often required in diagnostic and interventional procedures.
During an endovascular procedure, both the primary operator and the patient receive most of their $\mathrm{x}$-ray exposure from DSA acquisitions. ${ }^{3,4}$ The use of 3D DSA results in a significant decrease in the need to perform 2D DSA acquisitions. This reduction translated into a significant decrease in patient $\mathrm{x}$-ray exposure. ${ }^{5}$ While dose studies were not performed, it is estimated that the slight extension of acquisition time required for a 4D image acquisition (ie, 1 second) would result in an increase in radiation dose of approximately $20 \%$ compared with a conventional $3 \mathrm{D}$ acquisition. Because $3 \mathrm{D}$ image acquisitions represent, in general, only a minor fraction of the dose exposure during a conventional diagnostic or interventional procedure, this increase, when balanced against the potential reduc- 
Table 2: Raw data from each of the 2 observers for all 44 vessel segments $^{a}$

\begin{tabular}{|c|c|c|c|c|c|c|c|}
\hline \multicolumn{4}{|c|}{ Reviewer 1} & \multicolumn{4}{|c|}{ Reviewer 2} \\
\hline 2D & 3D & 4D & Preferred & 2D & 3D & 4D & Preferred \\
\hline 3 & 4 & 4 & $4 D$ & 2 & 4 & 4 & $4 D$ \\
\hline 3 & 3 & 4 & $4 D$ & 4 & 4 & 4 & $4 D$ \\
\hline 4 & 2 & 4 & $4 D$ & 0 & 3 & 4 & $4 D$ \\
\hline 4 & 2 & 4 & $2 \mathrm{D}$ & 3 & 4 & 4 & $4 D$ \\
\hline 4 & 2 & 4 & $4 D$ & 2 & 3 & 4 & $4 D$ \\
\hline 4 & 2 & 4 & $4 D$ & 3 & 3 & 4 & $4 D$ \\
\hline 4 & 3 & 4 & $2 \mathrm{D}$ & 3 & 4 & 4 & $4 D$ \\
\hline 4 & 3 & 3 & $2 \mathrm{D}$ & 4 & 2 & 3 & $4 \mathrm{D}$ \\
\hline 4 & 2 & 4 & $4 D$ & 4 & 3 & 3 & $4 D$ \\
\hline 4 & 4 & 4 & $4 D$ & 3 & 3 & 4 & $4 \mathrm{D}$ \\
\hline 4 & 4 & 4 & $4 D$ & 2 & 3 & 4 & $4 \mathrm{D}$ \\
\hline 4 & 4 & 4 & $4 D$ & 2 & 3 & 4 & $4 D$ \\
\hline 4 & 3 & 3 & $2 \mathrm{D}$ & 4 & 4 & 4 & $4 \mathrm{D}$ \\
\hline 4 & 3 & 4 & $2 \mathrm{D}$ & 2 & 4 & 4 & $4 D$ \\
\hline 4 & 3 & 4 & $4 D$ & 2 & 4 & 4 & $4 D$ \\
\hline 4 & 3 & 3 & $2 \mathrm{D}$ & 4 & 4 & 4 & $4 \mathrm{D}$ \\
\hline 4 & 4 & 2 & $3 D$ & 4 & 4 & 3 & $3 D$ \\
\hline 4 & 3 & 4 & $4 \mathrm{D}$ & 4 & 4 & 3 & $3 D$ \\
\hline 4 & 2 & 4 & $2 \mathrm{D}$ & 4 & 4 & 4 & $4 \mathrm{D}$ \\
\hline 4 & 1 & 3 & $4 D$ & 4 & 4 & 4 & $4 D$ \\
\hline 4 & 3 & 4 & $2 \mathrm{D}$ & 4 & 4 & 4 & $4 \mathrm{D}$ \\
\hline 4 & 4 & 4 & $2 \mathrm{D}$ & 4 & 4 & 4 & $4 D$ \\
\hline 4 & 3 & 4 & $2 D$ & 4 & 3 & 4 & $4 D$ \\
\hline 4 & 3 & 4 & $4 D$ & 4 & 4 & 4 & $4 D$ \\
\hline 4 & 4 & 4 & $2 D$ & 4 & 4 & 4 & $4 D$ \\
\hline 4 & 3 & 4 & $2 D$ & 4 & 3 & 3 & $2 D$ \\
\hline 3 & 2 & 2 & $2 \mathrm{D}$ & 2 & 0 & 1 & $2 D$ \\
\hline 4 & 4 & 4 & $4 D$ & 1 & 4 & 4 & $4 D$ \\
\hline 4 & 2 & 4 & $4 D$ & 2 & 2 & 4 & $4 D$ \\
\hline 4 & 3 & 4 & $4 D$ & 3 & 3 & 4 & $4 D$ \\
\hline 4 & 4 & 4 & $4 D$ & 4 & 3 & 4 & $4 D$ \\
\hline 4 & 4 & 4 & $4 D$ & 3 & 2 & 4 & $4 D$ \\
\hline 4 & 3 & 4 & $4 D$ & 2 & 2 & 4 & $4 D$ \\
\hline 4 & 4 & 4 & $4 D$ & 4 & 4 & 4 & $4 D$ \\
\hline 4 & 3 & 4 & $4 D$ & 3 & 3 & 4 & $4 D$ \\
\hline 3 & 3 & 4 & $4 D$ & 4 & 1 & 4 & $4 D$ \\
\hline 4 & 3 & 4 & $2 D$ & 3 & 4 & 4 & $4 D$ \\
\hline 4 & 2 & 4 & $4 D$ & 3 & 2 & 4 & $4 D$ \\
\hline 4 & 4 & 4 & $2 D$ & 4 & 4 & 4 & $4 D$ \\
\hline 4 & 3 & 4 & $2 D$ & 4 & 4 & 4 & $4 D$ \\
\hline 4 & 4 & 4 & $4 D$ & 3 & 4 & 4 & $4 D$ \\
\hline 4 & 3 & 4 & $4 D$ & 3 & 3 & 4 & $4 D$ \\
\hline 3 & 4 & 4 & $2 D$ & 3 & 4 & 2 & $4 D$ \\
\hline 4 & 4 & 3 & $2 D$ & 4 & 4 & 4 & $4 D$ \\
\hline
\end{tabular}

a The first 3 columns show the rating for each of the 3 modalities as described in the "Materials and Methods" and the scoring form (Fig 3B). The last column shows the preferred modality.

tion in exposure from 2D acquisitions is, in our opinion, acceptable.

This report represents a small series of animal studies and normal anatomy; we anticipate that the major advantages of $4 \mathrm{D}$ would be derived from the ability to delineate small-vessel abnormalities, particularly in areas where there is significant vascular overlap. In addition, more complex vascular anomalies with a significant flow-related component, such as arteriovenous malformations and arteriovenous fistulas, could be better evaluated because $4 \mathrm{D}$ allows for dissection of the angioarchitecture of early feeders and late venous drainage in several angles with 1 single acquisition.

The scales used for ranking the images and for the ratings of the display modes for the 4D-DSA images (MIP or surface-ren- dered) are qualitative. This feature combined with the sample size limited our ability to perform meaningful statistical evaluations. For this initial validation study, we, therefore, present the raw data. Both rating scales have been previously used and reported for similar image-evaluation tasks. ${ }^{2,6}$ The cranial arteries in the canine are smaller than those of humans. This, as well as their overlap, posed significant challenges for all of the tested modalities. The observation that 4D DSA was preferred as the technique to accomplish the given task of evaluating a series of vascular segments gives us confidence that the technique will be valuable if made available in a clinical environment. Future studies will include a larger patient population of human subjects with normal and abnormal vasculature to further demonstrate the potential applications of the technique.

\section{CONCLUSIONS}

In this study, 4D DSA was preferred over 2D and 3D DSA for analysis of normal vasculature. The ability to provide any view of a vascular territory at any time during passage of a contrast bolus seems likely to reduce the need for many 2D acquisitions during diagnostic and therapeutic procedures. This then potentially translates into a reduction in radiation and contrast dose.

Disclosures: Carolina Sandoval-Garcia-RELATED: Grant: National Institutes of Health, ${ }^{\star}$ Comments: study performed under institutionally approved National Institutes of Health R01 grant. Beverly Aagaard-Kienitz-RELATED: Grant: National Institutes of Health. * Sebastian Schafer-RELATED: Other: I am an employee of Siemens USA. Kevin Royalty_UNRELATED: Employment: I am a full-time employee of Siemens. Charles Strother-RELATED: Grant: Siemens, ${ }^{*}$ Comments: Imaging equipment and research support was provided under a Master Research Agreement between the University of Wisconsin Madison and Siemens; Support for Travel to Meetings for the Study or Other Purposes: support for travel to advisor meetings from Siemens under a Master Research Agreement between Siemens and University of Wisconsin School of Medicine and Public Health; Provision of Writing Assistance, Medicines, Equipment, or Administrative Support: Imaging equipment was provided under a Master Research Agreement between University of Wisconsin Madison and Siemens*; UNRELATED: Grants/Grants Pending: research support under a Master Research Agreement between University of Wisconsin Madison and Siemens*; Patents (planned, pending, or issued): I am coinventor on an issued patent for 4D DSA. Siemens has purchased a nonexclusive license for this intellectual property. * Money paid to the institution.

\section{REFERENCES}

1. Davis B, Royalty K, Kowarschik M, et al. 4D digital subtraction angiography: implementation and demonstration of feasibility. AJNR Am J Neuroradiol 2013;34:1914-21

2. Hinkmann FM, Voit HL, Anders K, et al. Ultra-fast carotid CTangiography: low versus standard volume contrast material protocol for a 128-slice CT-system. Invest Radiol 2009;44:257-64

3. Layton KF, Kallmes DF, Cloft HJ, et al. Radiation exposure to the primary operator during endovascular surgical neuroradiology procedures. AJNR Am J Neuroradiol 2006;27:742-43

4. Schueler BA, Kallmes DF, Cloft HJ. 3D cerebral angiography: radiation dose comparison with digital subtraction angiography. AJNR Am J Neuroradiol 2005;26:1898-901

5. Abe T, Hirohata M, Tanaka N, et al. Clinical benefits of rotational 3D angiography in endovascular treatment of ruptured cerebral aneurysm. AJNR Am J Neuroradiol 2002;23:686-88

6. Yu S, Yan L, Yao Y, et al. Noncontrast dynamic MRA in intracranial arteriovenous malformation (AVM), comparison with time of flight (TOF) and digital subtraction angiography (DSA). Magn Reson Imaging 2012;30:869-77 